\title{
Characterization of the polarization sensitivity anisotropy of a near-field probe using phase measurements
}

\author{
H. FISCHER, A. NESCI, G. LEVEQUE \& O.J.F. MARTIN \\ Nanophotonics and Metrology Laboratory, École Polytechnique Fédérale de Lausanne, 1015 \\ Lausanne, Switzerland
}

Key words. Green's tensor, heterodyne detection, nanoaperture, near-field probe, NSOM, phase measurement, plasmonics, polarization sensitivity, SNOM, surface plasmon.

\begin{abstract}
Summary
Amplitude and phase measurements of the near-field generated by isolated subwavelength apertures in a gold film are presented. The near-field distribution of such a structure is complex and the measured signal strongly depends on the electric field components effectively detected by the experimental setup. By comparing this signal with 3D vectorial calculations we are able to determine which electric field components are effectively measured. The sensitivity of the phase distribution is key to this measurement. The proposed characterization technique should prove extremely useful to calibrate a Scanning near-field optical microscopy (SNOM) beforehand in order to retrieve quantitative information on the polarization of the field distribution under study.
\end{abstract}

\section{Introduction}

Scanning near-field optical microscopy (SNOM) has become an important tool in a variety of scientific disciplines, in particular in nanophotonics and plasmonics, where the subwavelength resolution obtained with SNOM is essential to investigate fundamental phenomena and to develop devices with a subwavelength optical response. However, the images obtained with this technique are often difficult to interpret since different physical quantities can be measured depending on the SNOM configuration. These quantities include the intensity of the electric field (Pohl et al., 1984; Betzig et al., 1987), the amplitude and phase of the electric field (Balistreri et al., 2000; Nesci et al., 2001), the optical magnetic field (Devaux et al., 2000) or the local photon density of states (Chicanne et al., 2002). Furthermore the near-field probe itself and its motion along the sample surface can strongly influence the measured near-field, leading to a variety of artefacts (Hecht et al., 1997; Martin, 1999). Finally, every near-field probe has

Correspondence to: H. Fischer. Tel: 004121693 6843; fax: 004121693 2614; e-mail: Holger.fischer@epfl.ch. an anisotropic response, which records each component of the electric field differently (Labeke\& Barchiesi, 1993; Bozhevolnyi et al., 1999; Porto et al., 2000; Descrovi et al., 2005). This paper addresses this last issue: Using simultaneous measurements of the amplitude and phase of the near-field and comparing those with numerical simulations, we are able to determine which electric field components are effectively measured in our experiment. Another approach to characterize the response of near-field probes, relying on two-dimensional dielectric grating structures, has been proposed by Nesci et al. (2002).

In this paper individual subwavelength apertures in a gold film are used as sample. In addition to providing a well-defined - though complex - near-field distribution, those structures have attracted growing interest over the last years, since the discovery of enhanced optical transmission of light through arrays of subwavelength apertures in metallic films (Ebbesen et al., 1998; Genet \& Ebbesen). A variety of mechanisms have been brought forward to explain the extraordinary transmission observed in these systems. These mechanisms include surface plasmon polaritons (Martín-Moreno et al., 2001; Barnes et al., 2004), composite diffracted evanescent waves (Lezec \& Thio, 2004; Gay et al., 2006) and phase singularities (Schouten et al., 2004). It is obvious that the investigation of the optical transmission of light through a single aperture can further our understanding of enhanced transmission in aperture arrays. However, for a detailed analysis it is essential to have access to the full complex field. In this work heterodyne SNOM is used to measure simultaneously the amplitude and the phase of the near-field (Balistreri et al., 2001, 2000; Nesci et al., 2001). This technique has been used recently to measure amplitude and phase of propagating surface plasmon polaritons on structured (Offerhaus et al., 2005) and unstructured metallic surfaces, as well as the transmission of light through triangular aperture arrays (Nesci \& Martin, 2005). In the present paper the additional information provided by the measurement of the phase is used to determine which field components are effectively measured. 
To that extend, the measured data are compared with 3D calculations based on the Green's tensor technique .

The paper is organized as follows: The experimental setup and the simulation method are described in the next section. The results obtained from the simulation and from the experiments are compared and discussed in subsequent section. Finally a conclusion is given.

\section{Experimental setup and simulation technique}

The experimental setup used is as follows. A commercial SNOM (NT-MDT Ntegra) is mounted on an inverted optical microscope (Olympus IX71). The sample is illuminated at normal incidence from below with a $\lambda=532 \mathrm{~nm}$ wavelength laser through a low NA microscope objective (Olympus LMPLFLN5X), so that the illumination field can be considered as a plane wave. The sample is scanned with a near-field fibre probe in shear-force contact mode. The probe is coated with chromium and does not possess an aperture at the apex (Weeber et al., 1996). To simultaneously measure the amplitude and the phase of the near-field the SNOM is placed in one arm of a heterodyne interferometer (Balistreri et al., 2000; Nesci et al., 2001).

For the calculations the dyadic Green's tensor technique, based on the solution of the Lippmann-Schwinger equation for the electric field, is used (Martin \& Piller, 1998; Paulus et al., 2000; Paulus \& Martin, 2001). This method is well suited for the study of localized 3D objects embedded in a dielectric or metallic stratified medium. The value for the complex dielectric function $\varepsilon=\varepsilon^{\prime}+i \varepsilon^{\prime \prime}$ of gold has been taken from experimental tables (Johnson \& Christy, 1972) and interpolated to $\varepsilon^{\prime}=-5.6$ and $\varepsilon^{\prime \prime}=2.2$ for the illumination wavelength of $\lambda=532 \mathrm{~nm}$. The numerical fields plotted throughout the paper are calculated in a plane parallel to the sample surface (xy-plane) at a distance of $20 \mathrm{~nm}$ above the surface and for an incident planewave polarized in the y-direction incident from underneath the sample.

Note that the information on the phase and amplitude of the field allows the reconstruction of the time evolution of the fields. This is a crucial issue for the comparison of measured and simulated field maps, as will be discussed in the next section.

The sample is fabricated by focused ion beam milling (Nova 600 NanoLab) through a 150-nm-thick gold layer evaporated on a glass substrate with a 2-nm-thick chromium adhesion layer. Individual circular apertures with a 250-nm diameter are milled through the entire metallic film.

\section{Results and discussion}

Figure 1 shows measured xy-maps of the amplitude (a) and phase (b) of the electric field, as well as the topography for an individual circular aperture. In Fig. 1(c) the first-order plane has been subtracted from the original topographic data to obtain a flat image of the structure. Note that the diameter of the nanoaperture is too small for the SNOM tip
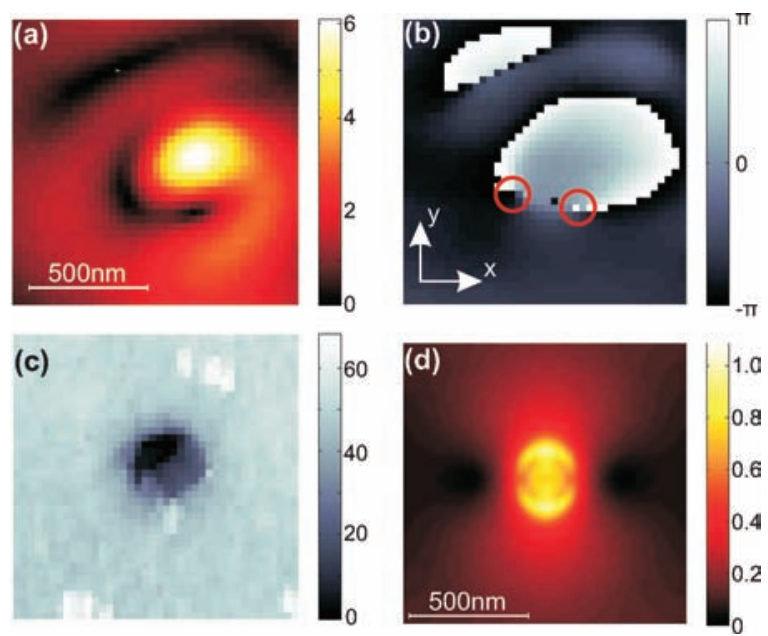

Fig. 1. Measured near-field amplitude [arb. units] (a), near-field phase [rad] (b) and topography [nm] (c) of an isolated circular subwavelength aperture. Two-phase singularities are marked in the measured phase pattern. (d) Calculated relative amplitude $20 \mathrm{~nm}$ above the surface.

to enter completely. Thus the measured aperture depth does not correspond to the film thickness. The amplitude of the normalized total electric field calculated for this structure is shown in Fig. 1(d). At first glance, a main difference is visible between the measured and calculated amplitude: The measured field exhibits one broad peak at the location of the aperture, Fig. 1(a), whereas the simulation shows two depolarization lobes, Fig. 1(d). These lobes have a simple physical origin: They occur at the rim of the aperture where the incident electric field is normal to the metal edge and a depolarization field is created to fulfil the boundary conditions imposed by Maxwell's equations (Martin \& Paulus, 2002). The distance between the two lobes in Fig. 1(d) (approx. $250 \mathrm{~nm}$ ) is such that the lobes should be well resolved with our SNOM. However, the experimental image shows just one somewhat distorted peak, Fig. 1(a).

The difference between measured and simulated images results from the complexity of the field detection mechanism in near-field microscopy. Specifically, the probe sensitivity to the various electric field components strongly depends on the tip used (Labeke \& Barchiesi, 1993; Bozhevolnyi et al., 1999). To investigate this in detail we calculate amplitude and phase maps for each electric field component $\mathrm{x}, \mathrm{y}$ and $\mathrm{z}$, as illustrated in Fig. 2. These results are in perfect agreement with those obtained by Ducourtieux et al. for subwavelength apertures in a chromium film (Grober et al., 1996; Ducourtieux et al., 2004). Very characteristic features are visible in the $\mathrm{x}$ - and z-components of the phase, namely lines of dislocation or singularity. When crossing such a line the phase undergoes a change of $\pi$, which means that the amplitude vanishes at the position of the singularity. For a point in space where the amplitude is zero the phase is not defined and thus singular (Nye, 1999). Since each electric field component 

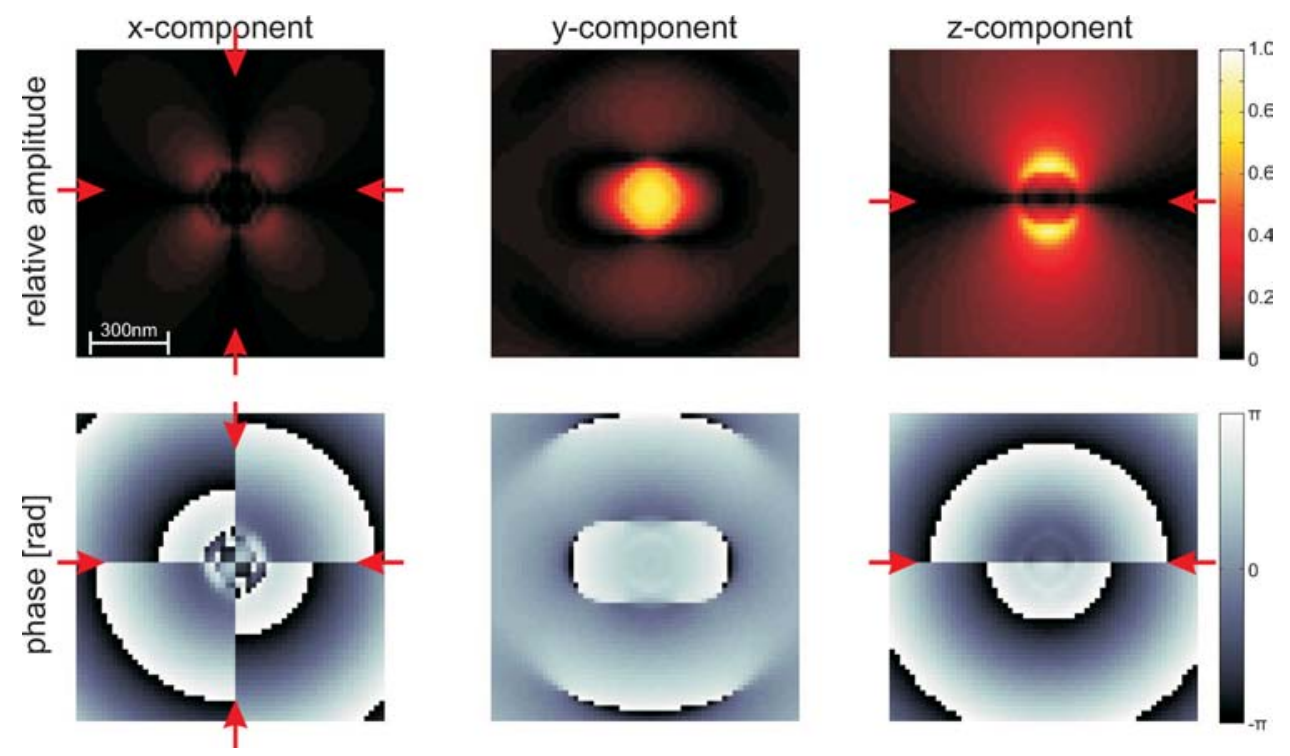

Fig. 2. Calculated components of the near-field amplitude and phase $20 \mathrm{~nm}$ above the sample surface. Lines of phase dislocation can be observed in the phase patterns for the $\mathrm{x}$ - and z-components (red arrows). At the corresponding lines the field amplitude is zero.

produces a very different phase distribution with specific features, it is possible to determine which field components are effectively measured by comparing measured and calculated phase maps. The direct measurement of phase distributions using heterodyne SNOM detection is key to this procedure.

Comparing the calculated (Fig. 2) and measured (Fig. 1(b)) phase distributions reveals obvious differences. For example, no dislocation lines are observed in the experimental data which rather exhibit two point dislocations marked with red circles in Fig. 1(b). Turning around these points the phase increases or decreases continuously (depending on the turning direction) so that the line integral over a closed loop is equal to $2 \pi$ (integration over a closed loop that does not include a phase dislocation gives zero) (Nye, 1999). In our experiment such screw dislocations were observed for every measured aperture. Furthermore, measurements in contact and constant height modes in the near-field zone have indicated that phase and amplitude maps did not change.

A simple comparison of experimental and calculated images clearly indicates that the near-field setup is not sensitive to one of the Cartesian $\mathrm{x}$, y or z field components, but to a combination of them. To determine the effectively measured polarization we combine different calculated components until we obtain a field distribution that resembles the experimental one. In this process, special attention is paid to the screw dislocations visible in the experimental data, Fig. 1(b).

A combination of the three electric field components can be characterized by the azimuth angle $\varphi$ and the polar angle (Fig. 3). The angle $\Theta$ determines the ratio of the $x-$ and $y$-polarizations, which can be combined into one field component parallel to the sample surface. The angle $\varphi$ determines the ratio between this parallel field component and the vertical (z-direction) field component. Any combination
$(\Theta, \varphi)$ of the field components leads to a different field distribution. In the following, we will concentrate on the ratio between the parallel and the vertical fields. As a matter of fact, assuming a circular symmetry along the z-axis for the probe, leads to an isotropic detection of the parallel fields and hence the possibility to choose $\varphi$ along one of the diagonals to mix equally the $\mathrm{x}$ - and $\mathrm{y}$-components. This results into two-phase maps mirror-inverted along the y-direction at the centre of the aperture. The best fit between the measured data, Fig. 1(b), and the computed data is obtained for $\Theta=-45^{\circ}$. It is important to mention that the heterodyne interferometer also influences the observed polarization since the polarization effectively measured corresponds to the projection of the recorded field onto the polarization of the field in the reference arm of the interferometer. This point is however beyond the scope of this paper and for the remaining of the discussion we will keep $\Theta$ constant and characterize the calculated field with the ratio $r=E_{\perp} / E_{\|}$between the perpendicular and the parallel field components.

In Fig. 3 the ratio $r$ is varied between $r=2$ and $r=0$. When the detected field is mainly vertical (Fig. 3(a), $r=2$ ) the field distribution very much resembles that of the purely vertical field shown in Fig. 2 (z-component). For $r=0.8$ a significant change is observed in the amplitude where the lower lobe disappears (Fig. 3(b), middle column). The maps calculated for $r=0.2$ show a very good resemblance with those measured, compare Fig. 3(c) with Figs. 1(a) and (b). For that ratio the dislocation line in the computed phase map transforms into two screw dislocations (marked with red circles in Fig. 3(c)) very similar to the measured pattern, Fig. 1(b). To highlight the resemblance of the measured and the calculated phase distributions a constant phase was added to each point of the measured map (Fig. 1(b)). Adding a constant phase to 
(a)
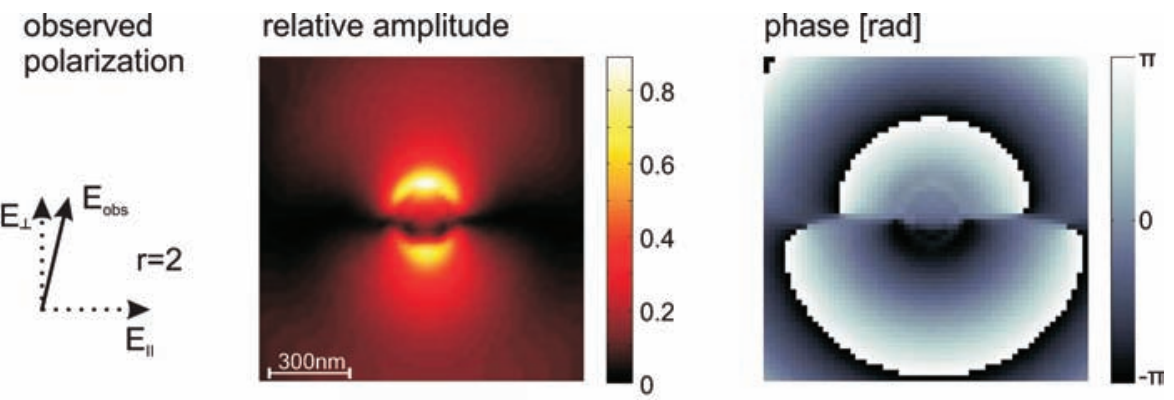

(b)
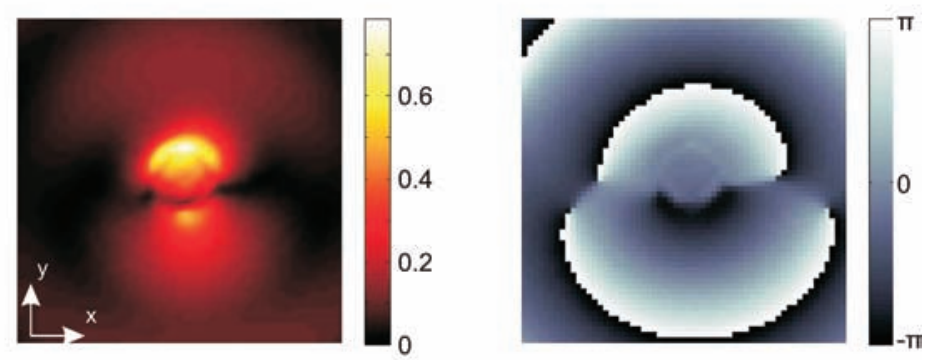

(c)<smiles>CCC(C)[C@H](C)[C@H](C)C(=O)O</smiles>
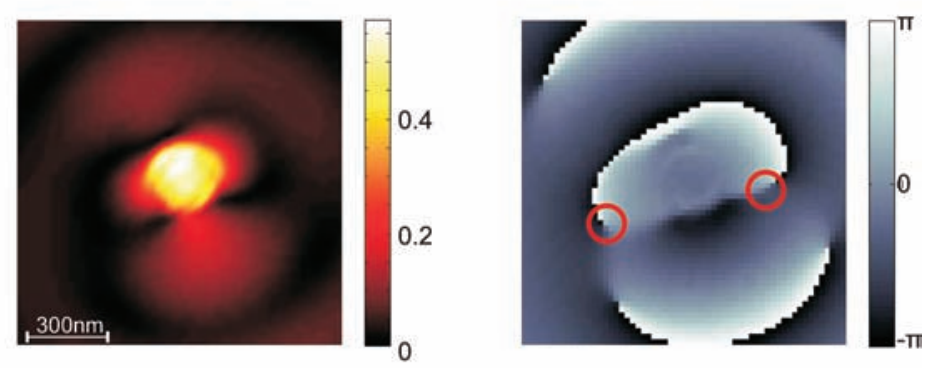

(d)
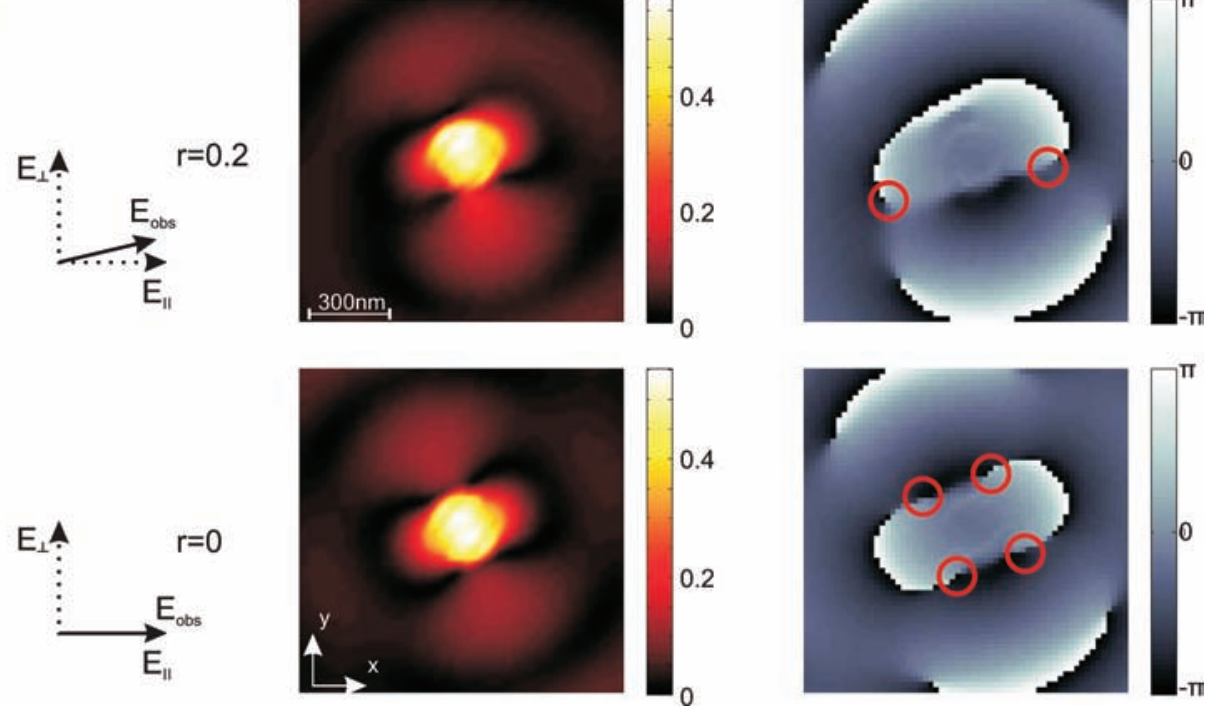

Fig. 3. Calculated amplitude and phase patterns $20 \mathrm{~nm}$ above the sample surface for different ratios $r=E_{\perp} / \mathrm{E}_{\mathrm{II}}$ for the observed polarization $E_{\text {obs }}$. For decreasing $r((\mathrm{a})-(\mathrm{d}))$ the dislocation line as observed for pure perpendicular polarization is converted into two-phase singularities as visible in the measured phase patterns. The strongest resemblance to the measurements can be found for $r=0.2$ (c).

the measurement is not a manipulation of the measured data but merely a shift in time. Indeed, measured and calculated maps are just snapshots in time and thus, for comparison, they need to be brought to the same time point. The calculated amplitude map at this ratio is also in good agreement with the measured amplitude map (compare Fig. 3(c) with Fig. 1(a)). For both figures, there is only one peak in the amplitude, at the location of the aperture. The slight rotation between measured and calculated phase maps results from the experimental setup where a perfect alignment between the polarization of the incident light and the y-direction of the scan is difficult to obtain.
Finally, for the detection of pure parallel polarization four phase dislocations are visible, $r=0$ (Fig. 3(d)). In this case, since the $y$-component of the field is much stronger than the $\mathrm{x}$ component (Fig. 2), the phase resembles mainly that of purely y-polarized field, but now turned by $45^{\circ}$ (Fig. 3(d)).

Although not shown here, let us emphasize that any other field combination investigated did not give such a good agreement between experimental and calculated data. Furthermore, measurements of several other apertures with the same probe have produced the same results. It is therefore reasonable to assume that the probe used in our measurement records the electric near-field with a sensitivity ratio between 
perpendicular and parallel polarizations in the order of $r=$ 0.2 .

\section{Conclusion}

In conclusion we have shown that near-field phase measurements provide important insights into the contrast mechanisms of SNOM. Characteristic features in the phase measurements, like screw or line dislocations, can be used to determine the field components effectively measured by a given probe. For the setup used in this work, the results indicate a sensitivity to the parallel field components that is about five times stronger than that to the perpendicular field components. This simple characterization technique should prove extremely useful to calibrate a SNOM beforehand in order to retrieve quantitative information on the polarization of the field distribution under study.

\section{Acknowledgement}

This work has been supported by the Swiss National Science Foundation

\section{References}

Balistreri, M.L.M., Gersen, H., Korterik, J.P., Kuipers, L. \& Hulst, N.F.V. (2001) Tracking femtosecond laser pulses in space and time. Science 294, 1080-1082.

Balistreri, M.L.M., Korterik, J.P., Kuipers, L. \& Hulst, N.F.V. (2000) Local observations of phase singularities in optical fields in waveguide structures. Phys. Rev. Lett. 85, 294-297.

Barnes, W.L., Murray, W.A., Dintinger, J., Devaux, E. \& Ebbesen, T.W. (2004) Surface plasmon polaritons and their role in the enhanced transmission of light through periodic arrays of subwavelength holes in a metal film. Phys. Rev. Lett. 92, 107401-107404.

Betzig, E., Isaacson, M. \& Lewis, A. (1987) Collection mode near-field scanning optical microscopy. Appl. Phys. Lett. 51, 2088-2090.

Bozhevolnyi, S.I., Vohnsen, B. \& Bozhevolnaya, E.A. (1999) Transfer functions in collection scanning near-field optical microscopy. Opt. Commun. 172, 171-179.

Chicanne, C., David, T., Quidant, R., et al. (2002) Imaging the local density of states of optical corrals. Phys. Rev. Letts. 88, 097401-097404.

Descrovi, E., Vaccaro, L., Aeschimann, L., Nakagawa, W., Staufer, U. \& Herzig, H.-P. (2005) Optical properties of microfabricated fullymetalcoated near-field probes in collection mode. J. Opt. Soc. Am. A 22, 1432-1441.

Devaux, E., Dereux, A., Bourillot, E., Weeber, J.-C., Lacroute, Y., Goudonnet, J.-P. \& Girard, C. (2000) Detection of the optical magnetic field by circular symmetry plasmons. Appl. Surface Sci. 164, 124-130.

Ducourtieux, S., Grésillon, S., Rivoal, J.C., Vannier, C., Bainier, C., Courjon, D. \& Cory, H. (2004) Imaging subwavelength holes in chromium films in scanning near-field optical microscopy. Comparison between experiments and calculation. Eur. Phys. J. Appl. Phys. 26, 3543.

Ebbesen, T.W., Lezec, H.J., Ghaemi, H.F., Thio, T. \& Wolff, P.A. (1998) Extraordinary optical transmission through sub-wavelength hole arrays. Nature 391, 667-669.
Gay, G., Alloschery, O., Lesegno, B.V.D., O’Dwyer, C., Weiner, J. \& Lezec, H.J. (2006) The optical response of nanostructured surfaces and the composite diffracted evanescent wave model. Nat. Phys. 2, 262-267.

Genet, C. \& Ebbesen, T.W. (2007) Light in tiny holes. Nature. 445, 39-46. Grober, R.D., Rutherford, T. \& Harris, T.D. (1996) Modal approximation for the electromagnetic field of a near-field optical probe. Appl. Opt. 35, 3488-3495.

Hecht, B., Bielefeldt, H., Inouye, Y., Pohl, D.W. \& Novotny, L. (1997) Facts and artifacts in near-field optical microscopy. J. Appl. Phys. 81, 24922498.

Johnson, P.B. \& Christy, R.W. (1972) Optical constants of the noble metals. Phys. Rev. B 6, 4370-4379.

Labeke, D.V. \& Barchiesi, D. (1993) Probes for scanning tunneling optical microscopy: a theoretical comparison. J. Opt. Soc. Am. A 10, 2193-2201.

Lezec, H.J. \& Thio, T. (2004) Diffracted evanescent wave model for enhanced and suppressed optical transmission through subwavelength hole arrays. Opt. Exp. 12, 3629-3651.

Martín-Moreno, L., García-Vidal, F.J., Lezec, H.J., Pellerin, K.M., Thio, T., Pendry, J.B. \& Ebbesen, T.W. (2001) Theory of extraordinary optical transmission through subwavelength hole arrays. Phys. Rev. Lett. 86, 1114-1117.

Martin, O.J.F. (1999) 3D simulations of the experimental signal measured in near-field optical microscopy. J. Microscopy 194 (pt. 2-3), 235-239.

Martin, O.J.F. \& Paulus, M. (2002) Influence of metal roughness on the near-field generated by an aperture/apertureless probe. J. Microscopy 205 (pt. 2), 147-152.

Martin, O.J.F. \& Piller, N.B. (1998) Electromagnetic scattering in polarizable backgrounds. Phys. Rev. E 58, 3909-3915.

Nesci, A., Dändliker, R. \& Herzig, H.P. (2001) Quantitative amplitude and phase measurement by use of a heterodyne scanning near-field optical microscope. Opt. Lett. 26, 208-210.

Nesci, A., Dandliker, R., Salt, M. \& Herzig, H.P. (2002) Measuring amplitude and phase distribution of fields generated by gratings with sub-wavelength resolution. Opt. Commun. 205, 229-238.

Nesci, A. \& Martin, O.J.F. (2005) Optical nano-imaging of metallic nanostructures. In: Plasmonic Nanoimaging and Nanofabrication. 1st edn. SPIE, San Diego, CA, USA.

Nye, J.F. (1999) Natural focusing and the fine structure of light. Bristol: Institute of Physics Publishing, Chapter 5.

Offerhaus, H.L., Bergen, B.V.D., Escalante, M., Segerink, F.B., Korterik, J.P. \& Hulst, N.F.V. (2005) Creating focused plasmons by noncollinear phasematching on functional gratings. Nano Lett. 5, 2144-2148.

Paulus, M., Gay-Balmaz, P. \& Martin, O.J.F. (2000) Accurate and efficient computation of the Green's tensor for stratified media. Phys. Rev. E 62, 5797-5807.

Paulus, M. \& Martin, O.J.F. (2001) Light propagation and scattering in stratified media: a Green's tensor approach. J. Optic. Soc. Am. A 18, 854-861.

Pohl, D.W., Denk, W. \& Lanz, M. (1984) Optical stethoscopy: image recording with resolution lambda/20. Appl. Phys. Lett. 44, 651-653.

Porto, J.A., Carminati, R. \& Greffet, J.-J. (2000) Theory of electromagnetic field imaging and spectroscopy in scanning near-field optical microscopy. J. Appl. Phys. 88, 4845-4850.

Schouten, H.F., Visser, T.D. \& Lenstra, D. (2004) Optical vortices near subwavelength structures. J. Opt. B 6, 404-409.

Weeber, J.-C., Bourillot, E., Dereux, A., Goudonnet, J.-P., Chen, Y. \& Girard, C. (1996) Observation of light confinement effects with a near-field optical microscope. Phys. Rev. Lett. 77, 5332-5335. 